\title{
TIC, MUJERES Y TranSICIÓN ESPAÑOLA ${ }^{1}$
}

\author{
Nieves Montesinos Sánchez \\ nieves.montesinos@ua.es \\ Universidad de Alicante
}

Recibido: 28-02-2013

Aceptado: 21-03-2013

\section{Resumen}

Este texto refleja claramente el contenido de nuestra investigación, ya que mediante y a través de ellas se consigue la transmisión del conocimiento y la recuperación de la memoria histórica en la Transición española. La hipótesis de partida del proyecto se centra en la existencia de un discurso sesgado de una época reciente y fundamental en la historia contemporánea, al haberse excluido, en términos generales a las mujeres de todo tipo de discurso. Con su desarrollo se conseguirá fortalecer una memoria colectiva cívica y democrática que incluya a los hombres y mujeres, introduciendo las vivencias, experiencias y discursos de estas últimas, mediante la creación de una herramienta hipermedia de gestión de contenidos (CMS) de investigación en soporte web.

Palabras Clave: Mujeres, web, Transición española, recuperación memoria cívica y colectiva.

\begin{abstract}
This paper clearly reflects the content of our investigation, once transmission of knowledge and recovery of Historical Memory in the Spanish Transition are obtained through it. This is based in the assumption that there is a biased report on a crucial moment of recent history, namely the Spanish Transition, since women have been, generally speaking, excluded from all kinds of discourses. Thus, a collective and civic memory, which includes both men and women, will be strengthened through the introduction of women's experiences and discourses. To that end, a research content management Hypermedia web tool (CMS) has been developed.
\end{abstract}

Keywords: Women, web, Spanish Transition, recovery of collective and civic memory.

\footnotetext{
${ }^{1}$ Este estudio ha sido realizado en el marco del Proyecto de investigación 'Haciendo Historia: Género y Transición en España', financiado por el MICINN (Ref. FEM2010-19068). La elaboración del mismo es fruto de la colaboración con Mar Esquembre y Carmen Mañas, integrantes del equipo de investigación del proyecto.
} 
- La historia, la solemne historia real, no me interesa casi nada. ¿Y a usted?

- Adoro la historia.

- ¡Qué envidia me da! He leído algo de historia, por obligación; pero no veo nada en ella que no me irrite o no me aburra: disputas entre papas y reyes, guerras o pestes en cada página, hombres que no valen gran cosa, y casi nada de mujeres, jes un fastidio!

(Jane Austen, citado por Dubby y Perrot, 2000: 33)

¿Qué se sabe de las mujeres? Las huellas que han dejado provienen menos de ellas mismas que de la mirada de los hombres que gobiernan la ciudad, construyen su memoria y administran sus archivos. El registro primario de lo que hacen y dicen está mediatizado por los criterios de selección de los escribas del poder. Y éstos, indiferentes al mundo privado, se mantienen apegados a lo público, un dominio en el que ellas no entran. Cuando irrumpen, entonces los escribas se inquietan como ante un desorden que [...] provoca idénticos estereotipos. [...] La relación entre los sexos deja su impronta en las fuentes de la historia y condiciona su densidad desigual.

(Georges Duby y Michelle Perrot, 2000: 33)

\section{Introducción}

"TIC, Mujeres y Transición española" refleja claramente el contenido de nuestra investigación y proyecto, ya que mediante y a través de ellas se contribuye a la transmisión del conocimiento y a la recuperación de la memoria histórica de las mujeres en la Transición española.

Aunque es relativamente reciente, los esfuerzos por la recuperación de la memoria histórica en nuestro país ${ }^{2}$, salvo excepciones, tienden en general a la recuperación para las generaciones actuales de la memoria de quienes

"directamente padecieron las injusticias y agravios producidos, por unos u otros motivos políticos
o ideológicos o de creencias religiosas [...] perdieron la vida [...] perdieron su libertad, al padecer
prisión, deportación, confiscación de sus bienes, trabajos forzosos o internamientos en campos de
concentración dentro o fuera de nuestras fronteras. [...] a quienes perdieron la patria al ser
empujados a un largo, desgarrador, y, en tantos casos, irreversible exilio [...]. Y a quienes en
distintos momentos lucharon por la defensa de los valores democráticos, como los integrantes del
Cuerpo de Carabineros, los brigadistas internacionales, los combatientes guerrilleros [...] o los
miembros de la Unión Militar Democrática, que se autodisolvió con la celebración de las primeras
elecciones democráticas" (España, 2007: 53410).

Con ello se contribuye, obviamente, y como reza la Exposición de Motivos de la Ley 52/2007, a "fomentar los valores constitucionales y promover el conocimiento y la reflexión sobre nuestro pasado, para evitar que se repitan situaciones de intolerancia y violación de los derechos humanos como las entonces vividas" (España, 2007: 53411).

\footnotetext{
${ }^{2}$ Cuyo máximo exponente a nivel estatal es la Ley 52/2007, de 26 de diciembre, por la que se reconocen y amplían derechos y se establecen medidas a favor de quienes padecieron persecución o violencia durante la guerra civil y la dictadura.
} 
Cabe hacer, al respecto, algunas consideraciones. En primer lugar, en las acciones de recuperación de la memoria histórica, ha de tenerse en cuenta que las experiencias de las personas a quienes hace referencia la ley poseen diferencias por razón de género, de un lado y, de otro, que las mujeres estaban excluidas de algunos ámbitos que gozan de primacía en el discurso historiográfico, como el bélico (Carabineros, brigadistas internacionales, guerrilleros, miembros de la U.M.D., etc.). Como es fácil comprobar, los instrumentos para la recuperación de la memoria (estudios, documentales, archivos históricos) tienden a invisibilizar a las mujeres.

En segundo lugar, la ley menciona a quienes "directamente padecieron las injusticias y agravios producidos, por unos $\mathrm{u}$ otros motivos políticos $\mathrm{o}$ ideológicos $\mathrm{o}$ de creencias religiosas", primando así una perspectiva individual que desdibuja, invisibiliza, oculta y silencia una dimensión colectiva: la de los motivos por razón de sexo. Se omite de esta forma que todas las mujeres, desde los inicios de la guerra civil, pero especialmente desde el fin de ésta y durante el franquismo sufrieron una brutal injusticia: la cercenación de su apenas estrenado estatuto jurídico pleno conquistado en la II República. Bajo la dictadura, es obvio, nadie goza de libertades, pero las mujeres quedarán, además, sometidas a un estatus secundario referido siempre a la tutela de un varón (padre, marido, hijo, consejo de familia), excluidas de cualquier ámbito y recluidas en la domesticidad. Si hay una característica básica del franquismo ésta es la de ser un sistema absolutamente patriarcal, legitimador y reforzador de estereotipos y roles diferentes para hombres y para mujeres, con subordinación de éstas a aquéllos.

En tercer lugar, al referirnos a la memoria histórica tendemos a identificarla con datos y hechos que se sitúan cronológicamente, casi de forma exclusiva, en la guerra civil y la dictadura franquista. Sin embargo, la estratificación social de género a que nos hemos referido y que encuentra su plena concordancia en los ámbitos jurídico y económico, está plenamente vigente no sólo durante el franquismo sino que se prolonga durante la transición española a la democracia, llegando hasta la actualidad en muchos aspectos.

\section{Justificación}

En el debate sobre las relaciones entre memoria e historia reciente (franquismo y transición) en España no se suele aludir más que de forma puntual a las experiencias femeninas. La ausencia de las mujeres en la construcción de nuestra memoria colectiva hace que ésta sea incompleta y sesgada. La memoria construye nuestra identidad, nos permite conocer nuestro pasado y contribuye a entender nuestro presente. Por eso resulta interesante reflexionar sobre qué y cómo se recuerda y qué se olvida sobre las mujeres en la memoria actual española, y la influencia que tiene esta memoria selectiva en la percepción del funcionamiento democrático de nuestro país. 
No obstante, el panorama es complejo. Como indica Josefina Cuesta (2008), existen distintos tipos de memoria, entre ellos, la memoria social -amplia, con contornos difusos- y la memoria colectiva, de un grupo más reducido, que está íntimamente relacionada con la memoria individual. La ausencia y el olvido de mujeres en la memoria actual de la sociedad española, tanto en la que podríamos identificar como memoria social como en la memoria colectiva, según la distinción citada, hace necesario rehabilitar las memorias femeninas.

Por ello, el proyecto se centra en recoger en formato audiovisual testimonios de mujeres que vivieron en el siglo XX y, concretamente, en la Transición. Las y los protagonistas de la historia tienen diferentes experiencias y desarrollaron distintas prácticas sociales en función de su identidad de género -entre otras variables, como la clase o la etnia- y las femeninas son menos conocidas. Este tipo de actuaciones planteadas en el presente proyecto permiten considerar a las mujeres como sujeto activo y no sólo como objeto de discurso, sino como actoras y agentes de cambio social y político.

Los testimonios audiovisuales resultan de gran importancia para el recuerdo de colectivos olvidados como el de las mujeres y para recoger experiencias, valoraciones y actitudes que no suelen aparecer en las fuentes escritas y, mucho menos todavía, audiovisuales. Las palabras de dirigentes políticas, integrantes de movimientos sociales y de mujeres anónimas tienen una presencia todavía poco destacada debido a su histórica dificultad de acceso a los medios de expresión y difusión.

Según Robert Frank,

"el testigo al que se solicita la memoria individual ofrece un testimonio revelador de una memoria colectiva, ella misma fruto de la interpenetración de diversas memorias y sufre la tensión entre la memoria de grupo -colectiva- y la memoria difusa-social-, entre la memoria oficial o histórica y la memoria común" (Frank, 1992: 69).

La recogida de testimonios de mujeres permite un enriquecimiento de la historia al completar el anterior vacío con una presencia femenina. Pero sobre todo hace posible replantear cuestiones y conceptos básicos como democracia, igualdad, ciudadanía, acción colectiva, militancia o identidad, que tradicionalmente se han interpretado en clave sólo masculina. De esta manera, podrían incluirse en los análisis históricos y políticos elementos como el acceso real, no sólo formal, a la ciudadanía política y social, la presencia desigual en los puestos de poder, la asimetría en las formas de militancia en función del género, el cuestionamiento de la división rígida entre lo público y lo privado, entre otros.

No se trata, sin embargo, en este proyecto de realizar una identificación entre testimonio/memoria y verdad histórica. Diversos autores han subrayado la diferencia entre memoria e historia. La memoria es reivindicación de un pasado y pretende legitimar, rehabilitar, honrar o condenar, mientras que la historia es un discurso construido, contrastable y objetivado, y busca conocer, interpretar o explicar.

De ahí que se advierta contra los abusos de la memoria (Cuesta Bustillo, 2008). Frente a dichos abusos, la investigación académica debe seguir avanzando en el conocimiento y, en no 
menor medida, contribuir a divulgar con seriedad las principales aportaciones científicas. Y eso es lo que se pretende con este proyecto a través del uso de las TIC.

La historiografía ha evolucionado hacia un creciente interés por los nuevos sujetos históricos, sus actitudes y valores, por lo que está introduciendo los análisis sobre las mujeres, proceso al que no es ajeno el desarrollo de la historia de género, muy atenta a la España del siglo XX. Por lo tanto, una aportación muy destacada de las investigaciones de género es integrar estas experiencias femeninas en el relato del pasado y replantear desde una perspectiva de género las interpretaciones de la historia española del siglo XX. Desde los años setenta, el desarrollo en España de los estudios de género en diversas disciplinas ha demostrado que el conocimiento del pasado, de la política y de cualquier ámbito de la sociedad se ha construido silenciando la voz y la actuación de las mujeres. Y si bien las aportaciones realizadas al respecto comienzan a ser bastante numerosas, no existe una buena síntesis que recoja las experiencias de las mujeres en la España del siglo XX y que incorpore los debates historiográficos más recientes, concretamente los referidos a la Transición española y los primeros años de nuestra democracia ${ }^{3}$. Las mujeres deben pasar de la periferia del discurso historiográfico y político a ocupar el centro, junto con los hombres. En suma, se trata de contribuir a una historia y a interpretaciones científicas alternativas, que integren voces olvidadas y tengan en cuenta nuevas perspectivas y que, en definitiva, contribuyan a un mejor conocimiento de nuestro pasado y de nuestra realidad actual.

En este sentido, en una sociedad de la imagen como la actual, aunque existen documentales interesantes sobre el protagonismo de las mujeres, en especial en la Guerra y la posguerra $^{4}$, sigue siendo muy necesario elaborar materiales audiovisuales y multimedia que ofrezcan nuevos elementos de reflexión, que aborden aspectos menos conocidos y, sobre todo, una visión de conjunto escrupulosa, que combine rigor académico y buena factura audiovisual, y que intente contextualizar y explicar las experiencias de las mujeres en la España del siglo $\mathrm{XX}$ en su etapa más reciente, que ya se sitúa entre el pasado y el presente, entre memoria e historiografía (Cuesta Bustillo, 2008: 417). De esta forma, se contribuye a un mayor y, sobre todo, un mejor conocimiento del pasado, que integrara voces y vivencias tradicionalmente relegadas en el discurso histórico, y a un presente que sepa afrontar con referencias sólidas los debates actuales.

\footnotetext{
${ }^{3}$ Las más recientes son las de Cuesta Bustillo (2003), con un enfoque con frecuencia sociológico, y Morant (2006), que abarca un ámbito cronológico y geográfico diferente.

4 "El siglo XX en femenino", del Institut de la Dona de la UV; "Nosotras que perdimos la paz", de Llum Quiñonero; "Mujeres en pie de guerra", de Susana Koska; "Los niños perdidos del franquismo", de TV3; "Abajo la dictadura" de la FEIS, entre otros. Un reciente documental se centra en las mujeres en la transición española, si bien limitado a las parlamentarias de la legislatura constituyente. Se trata de "Las Constituyentes" de Oliva Acosta (2011), el cual toma como punto de partida el estudio dirigido por Sevilla Merino (2006), del que una de las coordinadoras es Mar Esquembre.
} 


\section{Objetivos}

La hipótesis de partida del proyecto se centra en la existencia de un discurso sesgado de una época reciente y fundamental en la historia contemporánea, como es la transición española, al haberse excluido, en términos generales a las mujeres de todo tipo de discurso. El Proyecto de investigación en el que se encuadra el trabajo que presentamos, tiene tres objetivos generales prioritarios:

a) Fortalecer una memoria colectiva cívica y democrática que incluya a hombres y mujeres.

b) Fomentar la incorporación de la perspectiva de género en la investigación y en la docencia.

c) Creación de una herramienta hipermedia de gestión de contenidos (CMS) de investigación, en soporte web, para la consulta, difusión y generación de nueva investigación. El cumplimiento de los objetivos generales y prioritarios reseñados, se articula a través de los siguientes objetivos específicos: 1) Reconocer el contexto e incorporar el protagonismo social de las mujeres en los discursos historiográficos. 2) Establecer relaciones entre los discursos sobre las mujeres y las realidades vividas por ellas. 3) Poner en valor la posición social de las mujeres con la de los varones de su mismo grupo y compararla para deducir niveles de discriminación.

\section{Procedimiento}

La consecución de nuestros objetivos la planteamos mediante el desarrollo de una estrategia metodológica basada en el trabajo de campo, entendido este como un conjunto de acciones encaminadas a obtener de forma directa datos de las fuentes primarias de información, es decir, de las mujeres, y en el lugar y tiempo en el que se suscita el conjunto de hechos de interés para el objeto de la investigación. Para llevar a cabo este trabajo de campo, con el máximo rigor es necesario, tal y como indica Sabino (1995), diseñar previamente las secuencias a seguir con la finalidad de poder contrastar de la mejor manera posible las condiciones en las que se han obtenido los datos. Por otra parte, este tipo de estrategia metodológica nos permite combinar distintos diseños de recogida de información, sistemática y fiable. Para comenzar a trabajar sobre nuestro primer objetivo específico: Fortalecer una memoria colectiva cívica y democrática que incluya a hombres y mujeres, es imprescindible conocer las experiencias vividas por las mujeres españolas en el pasado reciente:

Aproximación al contexto de la investigación: La Segunda República (1931-1936), el primer régimen democrático de la historia de España, despertó importantes expectativas entre muchas mujeres. El estallido de la Guerra Civil (1936-1939) supuso, en el bando republicano, 
la aparición de las milicianas que acudían al frente. Su imagen de mujeres activas y con el fusil en la mano produjo un gran impacto. Con la victoria del ejército rebelde, se implantó la Dictadura franquista (1939-1975), que se convirtió en una doble dictadura para las mujeres: por la pérdida de derechos políticos, sociales y económicos, y por la vuelta a la discriminación en los códigos penal y civil, que las convirtió en menores de edad, dependientes de sus padres o maridos. Las dos instituciones que encuadraron a las mujeres fueron la Sección Femenina de FET y JONS, que desarrolló tareas de adoctrinamiento político y de género, a través de las clases de educación física, el Servicio Social o el Auxilio Social, y la Iglesia católica, omnipresente en todo acto público o privado, que impuso una moral rigorista y muy exigente a las españolas. A pesar de ello la resistencia de las mujeres se organizó y de su lucha durante los años cuarenta y cincuenta, surgió en 1965 el Movimiento Democrático de Mujeres (MDM), vinculado al PCE. El feminismo fue reorganizándose también a partir de las publicaciones de intelectuales católicas o de izquierda como María Laffitte, Lilí Álvarez, María Aurelia Capmany o Lidia Falcón, quienes escribieron sobre la situación de las mujeres en España en libros y revistas. En el mismo sentido, fue importante la aparición de algunas organizaciones pioneras, como la Asociación de Mujeres Universitarias o el Seminario de Estudios Sociológicos sobre la Mujer.

Uno de los primeros resultados de nuestra investigación se centra en la constatación de la insuficiencia del mero aporte de datos omitidos u olvidados en la historiografía tradicional para la ampliación de la historia de la transición de tal manera que incluya la necesaria perspectiva de género. Como acertadamente afirma Mónica Threlfall (2009: 17),

"la mayor parte de la investigación sobre la transición a la democracia ha mantenido el silencio sobre las cuestiones de género, sean éstas las actividades de las organizaciones de mujeres, las protestas contra el sexismo del régimen de Franco, o los temas de igualdad en la Constitución, el debate parlamentario y los partidos políticos".

Aunque ya hay investigaciones en el ámbito de los estudios feministas y de género relativas a este periodo histórico reciente, el reto se centra ahora en ofrecer una visión de conjunto que permita concluir que "sin eso, sin el feminismo, la historia de la democratización hubiera sido otra" (Threlfall, 2009: 17 y 44). Esta tarea la hemos comenzado con la exposición "Mujeres de la Transición". En ella, partiendo de la aproximación al contexto a la que nos hemos referido con anterioridad, se refleja, en líneas generales y de forma muy visual, la participación y el papel activo y decisivo de las mujeres y de los movimientos feministas en el proceso de cambio hacia la democracia en todos los ámbitos y no sólo en el de las elites en el ámbito público. Así, en los años sesenta y setenta se experimentaron importantes cambios en la situación de las mujeres en España, provocados por la modernización de la economía, la

\footnotetext{
${ }^{5}$ Museo de la Universidad de Alicante (MUA), marzo 2011. Moreno, Mónica; Esquembre, Mar; Establier, Helena; Mañas, Carmen; Montesinos, Nieves (2012). Catálogo Mujeres de la Transición. Alicante: Museo de la Universidad de Alicante. En él la autoría de los textos corresponde a Mónica Moreno y a Mar Esquembre. A la Exposición acompaña un audiovisual (Mariló Berenguer. FGUA).
} 
ampliación del sistema educativo y el comienzo de un cambio en la moral y las costumbres donde el papel del movimiento de mujeres no es desdeñable. Las mujeres empezaron a vestir y a comportarse de una manera menos encorsetada, y las jóvenes, sobre todo, accedieron a los pantalones y minifaldas, al tabaco y los coches.

El desarrollismo provocó un fuerte éxodo rural y el crecimiento rápido de las ciudades y con ellas la aparición de los barrios nuevos que carecían de infraestructuras y servicios necesarios y las mujeres que vivían en esos barrios participaron, de forma contundente, en la movilización vecinal cuyas organizaciones se caracterizaron por crear Vocalías de Mujeres que contribuyeron a la difusión de principios feministas. Por otro lado las asociaciones de amas de casa y consumidores, cuya trayectoria sigue viva en nuestros días, a pesar de haberse creado al amparo de la ideología de la dictadura, pronto evolucionaron hacia planteamientos feministas, reivindicando igualdad entre hombres y mujeres y el acceso al trabajo remunerado en igualdad de condiciones, algo que todavía hoy no hemos conseguido.

Este cambio económico requería una extensión y mejora del sistema educativo. La participación de muchas jóvenes en las protestas estudiantiles de la transición, tanto en las universidades como en los institutos de secundaria, significó no solo intervenir en la demanda de un funcionamiento democrático de las instituciones educativas, sino también un activismo en contra de la dictadura y a favor de las libertades. En los grupos de oposición estudiantil, con frecuencia vinculados al PCE o a otros partidos de izquierda, las mujeres asumieron actividades arriesgadas pero poco reconocidas, como el reparto de propaganda.

De forma paralela, se fue gestando un movimiento de enseñantes que impulsó la renovación pedagógica y exigió el establecimiento de una escuela participativa, pública, laica y coeducativa. Otras profesionales destacaron también en la lucha antifranquista. Muchas abogadas defendieron los derechos laborales de trabajadores y trabajadoras, ofrecieron asesoría jurídica en barrios populares de las grandes ciudades o defendieron a personas encarceladas por su actividad política de oposición a la dictadura. Desde los medios de comunicación, las periodistas contrarias al franquismo sortearon la censura e informaron sobre las movilizaciones políticas, sindicales o cívicas de la transición, contribuyendo a crear un estado de opinión favorable al nuevo régimen democrático.

La cultura durante la Transición se convirtió en un espacio más de lucha antifranquista, en defensa de las libertades democráticas, del respeto a la pluralidad nacional y de nuevas formas de expresión artística. Entre las artes que más destacaron en este sentido, cabe mencionar la música, con el auge de la canción protesta, a la que pertenecieron solistas de la talla de Ana Belén, Rosa León o Elisa Serna, entre otras. Además, en los territorios de habla catalana y en general en todo el estado español, la nova cançó adquirió una gran popularidad, con voces como la de María del Mar Bonet. Ya a principios de los ochenta, a medida que el régimen democrático fue consolidándose, aparecieron nuevas propuestas musicales transgresoras y críticas con la moral tradicional, como el grupo femenino punk Las Vulpes. También desde las artes plásticas, el teatro, o el cine numerosas mujeres manifestaron su crítica a la dictadura y su apoyo a la democracia, contribuyendo a la difusión de estos principios y al 
desarrollo de una cultura y una sociedad más libres. Otras mujeres, anónimas o menos conocidas, participaron en las diversas manifestaciones culturales populares que proliferaron en la transición. También las escritoras se incorporaron con fuerza al mercado editorial español, que asistía a un auténtico "boom" de la literatura escrita por mujeres, especialmente en el ámbito de la narrativa. En estos años publicaron sus primeras novelas Esther Tusquets, Rosa Montero, Marina Mayoral, Soledad Puértolas y Carme Riera, entre muchas otras. Por otra parte, surgieron iniciativas vinculadas al feminismo como la editorial LASal o las librerías de mujeres, que impulsaron una cultura en femenino.

La celebración en 1975 del Año Internacional de la Mujer, convocado por la ONU, permitió que tuvieran lugar las I Jornadas de Liberación de la Mujer en Madrid. Con ellas, los movimientos de mujeres intentaron demostrar la existencia de una realidad paralela a la oficial Sección Femenina, ya en crisis. Las organizaciones feministas más importantes fueron el MDM (Movimiento Democrático de la Mujer), de composición plural pero vinculada al PCE, la ADM (Asociación Democrática de la Mujer, denominada Associació Catalana de la Dona en Cataluña), relacionada con el PTE, y el Partido Feminista, entre muchas otras. Se agruparon en diversas asambleas, federaciones y coordinadoras, en torno a distintos planteamientos que dieron lugar a un rico debate feminista: feminismo de la igualdad (que insistía en la consecución de la igualdad entre mujeres y hombres) o feminismo de la diferencia (que reivindicaba principios asignados a las mujeres, como la afectividad o la sensibilidad, y revalorizaba la identidad femenina); partidarias de la militancia única (feminista) o de la doble militancia (en partidos y en asociaciones de mujeres); feminismo socialista, radical o de la tercera vía. Estas posiciones afloraron en nuevas jornadas, como las que tuvieron lugar en Barcelona (1976), Valencia (1977) o Granada (1979). A partir de esa fecha y de la consolidación de la democracia, el feminismo entró en una fase de institucionalización. Desde esa base institucional se lanzaron campañas de sensibilización sobre las reivindicaciones feministas. El derecho al divorcio y la despenalización del aborto, fueron las campañas más controvertidas y las que más impacto mediático tuvieron, por las connotaciones políticas, morales y religiosas que adquirieron. Sobre la despenalización del aborto, las feministas reclamaban, una vez más, el derecho de las mujeres a decidir sobre sus vidas, con lemas como "nosotras parimos, nosotras decidimos", si bien el aborto era considerado un atentado contra el cuerpo de las mujeres y último recurso ante una maternidad no deseada. En esta larga campaña hubo hitos muy sonados, como los actos de solidaridad con once mujeres juzgadas en Bilbao acusadas de abortar o haber ayudado a ello, o las recogidas de documentos de autoinculpación ("yo también he abortado"). En 1985 se consiguió la despenalización de la interrupción voluntaria del embarazo pero solo en tres casos (eugenésico, como resultado de una violación y social). Hoy el tema del aborto sigue estando en el debate.

Otras campañas para conseguir unas relaciones para mujeres y hombres igualitarias y respetuosas fueron: la doble jornada laboral y la doble explotación económica de las mujeres. La demanda de una educación coeducativa, y laica. El derecho a decidir sobre la vivencia sexual de cada quien y el respeto al lesbianismo, denunciando la doble discriminación de las 
homosexuales por mujeres y por lesbianas. La denuncia de la violencia contra las mujeres fue otro de sus objetivos. Revistas y manifestaciones criticaron los malos tratos y las violaciones como resultado de unas relaciones de género desiguales.

Junto a la movilización social y feminista, la presencia de las mujeres fue muy destacada en la dinámica actividad política de la transición, aunque pocas veces ostentaron cargos de representación Aunque la presencia de las mujeres fue muy destacada en la dinámica actividad política de la transición, su histórica exclusión de todos los ámbitos de la vida social también se haría patente en la conformación de las candidaturas electorales, requisito para el acceso a la representación política. Si la presencia de las mujeres en las candidaturas electorales constituía un porcentaje mínimo, éste se redujo prácticamente a la mitad en los resultados de las elecciones. Las mujeres en las Cámaras legislativas eran casi una anécdota. Sólo 21 escaños de los 350 del Congreso de los Diputados fueron ocupados por mujeres en la Legislatura constituyente, lo que representaba el 6\% de la Cámara. En el Senado ni siquiera llegaban al $2 \%$, pues junto a las dos senadoras de designación real sólo cuatro mujeres resultaron electas, ocupando así 6 asientos de los 248 que conformaban la Cámara alta.

Tampoco en el Gobierno hubo mujeres. Salvo el caso de Soledad Becerril, nombrada Ministra de Cultura en 1981, a punto de finalizar el mandato, ninguna mujer volverá a ocupar una cartera ministerial hasta 1988.

No muy diferente fue la situación en los Ayuntamientos y en las Comunidades Autónomas.

La exposición se acompaña de un audiovisual con fragmentos seleccionados de algunas de nuestras entrevistadas que, con sus testimonios, tejen el relato de esa otra historia silenciada pero sin la que nuestra democracia hubiera sido otra y de peor calidad ${ }^{6}$.

\section{Conclusiones}

Los resultados obtenidos hasta el momento en nuestra investigación ponen de manifiesto la necesidad de poner en valor el papel de las mujeres en este periodo que nos ocupa, ya que como podemos deducir, muchas de las campañas e iniciativas puestas en marcha en esos años siguen hoy vivas, es decir sujetas a debate, y pese a que muchas de las cuestiones defendidas por las mujeres han entrado en la agenda política, siguen sin ser consideradas prioritarias.

El proyecto está en la fase de recogida de testimonios mediante la realización de entrevistas a las protagonistas, y al tiempo, conseguimos todos aquellos documentos que puedan enriquecer las entrevistas para digitalizarlos y que formen parte de la memoria recogida. Los contenidos se guardan en una base de datos para la exportación, catalogación o

\footnotetext{
${ }^{6}$ Tanto la Exposición como el audiovisual pueden verse en: http://www.mujeresytransicion.es
} 
búsqueda. Y puesto que la mejor manera, entendemos, de transferir y fomentar el conocimiento es ponerlo a disposición, el resultado final estará en formato web para su difusión vía Internet. La clave para la accesibilidad a los testimonios, documentos e investigaciones y estudios, es que el sistema se basa en un gestor de contenidos hipermedia con diseño web.

\section{BIBLIOGRAFÍA}

- Cuesta Bustillo, Josefina (2003): Historia de las mujeres en España. Siglo XX. Instituto de la Mujer: Madrid.

. (2008): La odisea de la memoria. Historia de la memoria en España. Siglo XX. Madrid: Alianza Editorial.

- Duby, Georges y Perrot, Michelle (2000): "Escribir la historia de las mujeres". En: Georges Duby y Michelle Perrot (dirs.): Historia de las mujeres, vol 1: La Antigüedad. Madrid: Taurus, pp. 21-33.

- España (2007): "Ley 52/2007, de 26 de diciembre, por la que se reconocen y amplían derechos y se establecen medidas a favor de quienes padecieron persecución o violencia durante la guerra civil y la dictadura”. En: Boletín Oficial del Estado, $n^{\circ} 310$, de 27 de diciembre de 2007, pp. 53410-53416, [en línea] Disponible en: http://www.memoriahistorica.gob.es/NR/rdonlyres/05A8266F-E313-4785-A5D7-3F32E11B1 2AC/0/PublicacionLeyBoe.PDF [12/01/2013].

- Frank, Robert (1992): “La mémoire et l'histoire". En: Danièle Woldman (dir.) : La bouche de la vérité? La recherche historique et les sources orales, número monográfico de Cahiers de l'IHTP, $\mathrm{n}^{\mathrm{o}} 21$, pp. 65-73.

- Morant, Isabel (2006): Historia de las mujeres en España y América Latina. Madrid: Cátedra.

- Sabino, Carlos A. (1995): El proceso de investigación, 2a ed. Bogotá: Editorial Panamericana.

- Threlfall, Mónica (2009): "El papel transformador del movimiento de mujeres en la transición política española”. En: Carmen Martínez Ten; Purificación Gutiérrez López y Pilar González Ruiz (eds.): El movimiento feminista en España en los años 70. Madrid: Ed. Cátedra, col. Feminismos, pp.17-52. 